\title{
The contributions of 3D digital printing to Dentofacial Orthopedics
}

\author{
B. Roger ${ }^{1}$, J.-B. Roger ${ }^{2}$ \\ 1 Specialist in Dentofacial Orthopedics, private practice \\ 2 Student at the School of IPSO, Isabelle Dutel Academy of Dentistry, Paris
}

\section{SUMMARY}

When a digital impression is created, practitioners tend to get excited about finding the best approach to use it. The 3D printer obviously comes at the end of the virtual sequence of materialization of an object from a digital file.

It's a disruptive innovation [Campbell T. et al. Could 3D Printing Change the World? Atlantic Council Reports 2011.] that can also improve the therapeutic procedure.

KEYWORDS

Digital impression, 3D printing, digital workflow

\section{INTRODUCTION}

The technological evolution has allowed our specialty to evolve over time. The digital world has invaded our daily lives, and 8-number series of zeros and ones called bytes are being used for the binary coding of various types of information. These range from the simplest to the most complex information, including images, sounds, and also object specifications.

When developing the typographical apparatus to print the Bible back in 1454, Gutenberg did not think that many years later, machines would allow everyone to personally edit a text, either at home or remotely. Such printing leaves ink traces on sheets of paper, which is geometrically a two-dimensional (2D) plane.

Three-dimensional (3D) printing is derived from this notion but is achieved by superimposing these planes. The easiest way to grasp this concept is the orthonormal frame, but there are other more complex frames of reference such as the polar and angular coordinate systems.

Strictly speaking, 3D printers are machines that permits the additive construction of a volume digitized in bytes ${ }^{3}$.

The prospect offered by this achievement is of particular interest because of the many possibilities that it affords for cast construction and equipment fabrication.

\section{Address for correspondence:}




\section{THE WORK BASE: OPTICAL IMPRESSIONS}

Digitization is a theme that has been discussed in detail throughout this issue, but we will only reiterate that there are two ways of digitizing the volumes of each arch.

The intraoral scanner allows us to directly digitize the inside of the mouth. According to the material used $4,5,10,12,17$, the result of this scan is in the format of an STL file (stereolithography). This file format makes it possible to characterize the outer surface of an object using a series of triangles. It is readable and freely decipherable by nonproprietary software.

According to the manufacturers, the precision of the "optical impression" varies considerably but can easily reach about 10 microns.

The tabletop scanner can digitize plaster models or can be applied directly onto the impression. In this case, in terms of precision, we will add the defects of the classical model to that of the optical impression to create the STL file. This STL file will not need to be transported to the lab via a motorized courier because an Internet connection would suffice. However, a good connection is required as the file is large. The software provided by the manufacturers makes it easy to send the file and to communicate with orthodontists via email. Considering that the volume of data to be saved is substantial, a cloud-type data host can also be used. In any event, a secure connection is mandated as the subject matter concerns personal medical data. The legislators seem to have thought of this problem by giving specific approval from the Ministry of Health to these data-hosting sites.

\section{D PRINTING}

Because 3D printing is an additive manufacturing technology, the principle of construction lies in the superposition of different layers of material that will ultimately form the object to be materialized.

If the object is placed on a direct orthonormal frame $(\mathrm{O}, \mathrm{x}, \mathrm{y}$, and $\mathrm{z})$, the successive layers will be on the $(0, x$, and y) plane along the z-axis. In practice, our printing base, which is the plate, will, therefore, have a translation movement along the z-axis. The process is the same for all the different printing technologies, from wireframe to laser sintering and stereolithography. The plate will evolve along its axis according to the pitch determined by the desired precision. According to this principle, there are different technologies at our disposal to respond to all our needs. The most widespread technology used by the general public is fused deposition modeling. It should be noted that the quality of the result is poor and does not allow the printing of a finished product, and its use on working models is not synonymous with a quality result. There are multiple printable materials - most of the thermoplastic polymers such as ABS, polycarbonate, and polyethylene terephthalate (PET), can be used. 
If we want to be able to print quality finished parts or precision working or study models, a technique that is much more adapted to the dental world-stereolithography or SLA-is preferred ${ }^{6,7}$. The material used here is not to be confused with thermoplastic; instead, it is a photopolymerizable liquid resin. According to the principle of additive manufacturing, the printing plate will be immersed in a tray containing the liquid resin and a beam of light will be projected; this will activate the photoinitiators.

The light source will have a precise main wavelength, often between 300 and $450 \mathrm{~nm}$, and may come from a source such as a laser, which will scan the area to be polymerized. Alternatively, it may be as simple as projecting the image of the layer concerned from a video projector whose source will be adapted to polymerize the resin.

Existing materials allow us to print a fairly-wide range of elements, including, but not limited to, the following: resins for study and working models, calcinable resin, transparent biocompatible resins with different mechanical properties, resin for temporary crowns, flexible gingiva or even custom tray resin.

The availability of SLA printers is rather varied, but there are large disparities in reliability. Unfortunately, the entry-level models seem too unreliable to be able to use them in a secure and professional way. Nevertheless, they allow us to apply the technique.

The technique that directly rivals SLA is Polyjet; 9,16 a photopolymerizable resin is used once again but will be sprayed in fine droplets on the printing plate and then polymerized. The main advantage is that it is possible to project different resins at the same time.

Each printed part is maintained by a support wax printed at the same time. This wax is then eliminated, either in a suitable bath or in an oven. The part released from this support wax is thus ideally printed and left without a trace of support, which is not possible in SLA. The printing supports are necessarily made of the same material as the finished part, which therefore requires the manual removal of these supports.

The latest possible technology is an industrial solution. To date there has been no record of solutions made accessible in a simple orthodontics laboratory using laser sintering or selective laser melting $(\mathrm{SLM})^{16}$. Basically, metal parts will be made from the powder of the chosen metal: titanium, stainless steel, or aluminum. This provides numerous possibilities. A powerful fiber optic laser is used to agglomerate the powdered metal particles. This technique is also applicable to ceramics; in fact, many people are currently using it in this way.

Why not consider using this technique to create individualized ceramic locks (or latches).

\section{USING THE DIGITAL IMPRESSION}

In theory, the digital age has given us reproducibility and precision as long as we make the best use of the tech- nologies available. It should be noted that cumulative inaccuracies can be significant even when working digitally. 
To compare the various inaccuracies that are apparent, the following steps should be followed. Firstly, we will position ourselves in a one-dimensional space to facilitate the illustration. It will then suffice to extrapolate the procedure used in the other two spatial dimensions to appreciate the actual inaccuracy.

Let us consider that when scanning a point on a tooth to be digitized, every step of the process from the transformation, digitization, design, fabrication, and post-processing will lead to some inaccuracy. We associate the numerical value in microns of the latter with the value of an angle in degrees which, in a completely arbitrary manner, will geometrically represent the inaccuracy of a given step. That being said, a step $n$, with a precision of $\geq 50$ microns, will then be associated with a $50^{\circ}$ angle.

Step $n+1$ will likewise generate an angle which will be placed geometrically at the two ends of the angular sector of step $n$. The final opening created by this suite will simulate the inaccuracy. We will compare the two different openings obtained to show, in relative terms, the benefit of a continuous digital flow.

Let us also include the examples of a traditional impression then a plaster cast and finally a scan using a tabletop scanner. The inaccuracy values are average.

The assumed numerical value of the final opening is 100 . The numerical value calculations we performed using a mechanical design software. (fig. 1).

Let us now consider the case where the impression, digital design, and additive manufacturing product are scanned. The opening is decreased by half (figure 2).
Finally, in continuous digital flow, an intraoral scan is performed and the apparatus is designed in a manner similar to the two previous cases. The size of the opening drops to 20; the imprecision is divided by five (fig. 3 ).

By taking these data into account, we can make an informed use of our digital impression. Its first use is the direct printing of study models. For printing study models, alginate and plaster are suitable. The investment cost for optical impressions are too high for this use alone. It should therefore only be used for another more adapted purpose because the solidity of the resin allows it to resist any mistreatment or handling error. The wired printer also provides an acceptable result for this particular task.

For orthodontists, the digital impressions are working models in which the bands can be mechanically adjusted (according to the proposed treatment plan) and attachments can be positioned and thermoformed. This achieves nothing more than the plaster models.

The condition for success is the proper programming of the printer to obtain an accurate dimensional rendering.

The type of innovation afforded by the digital model is manifested in the ability to directly manufacture devices (known as computer-aided design). This involves the use by the orthodontist of a design software to virtually build what the practitioner has prescribed, and then move on to additively construct it using a 3D printer, which in this case will be of the stereolithographic kind.

Depending on the specificities of each machine, software is used to position the part or parts to be printed on the tray. It is possible to choose (depending on whether optimizing the 


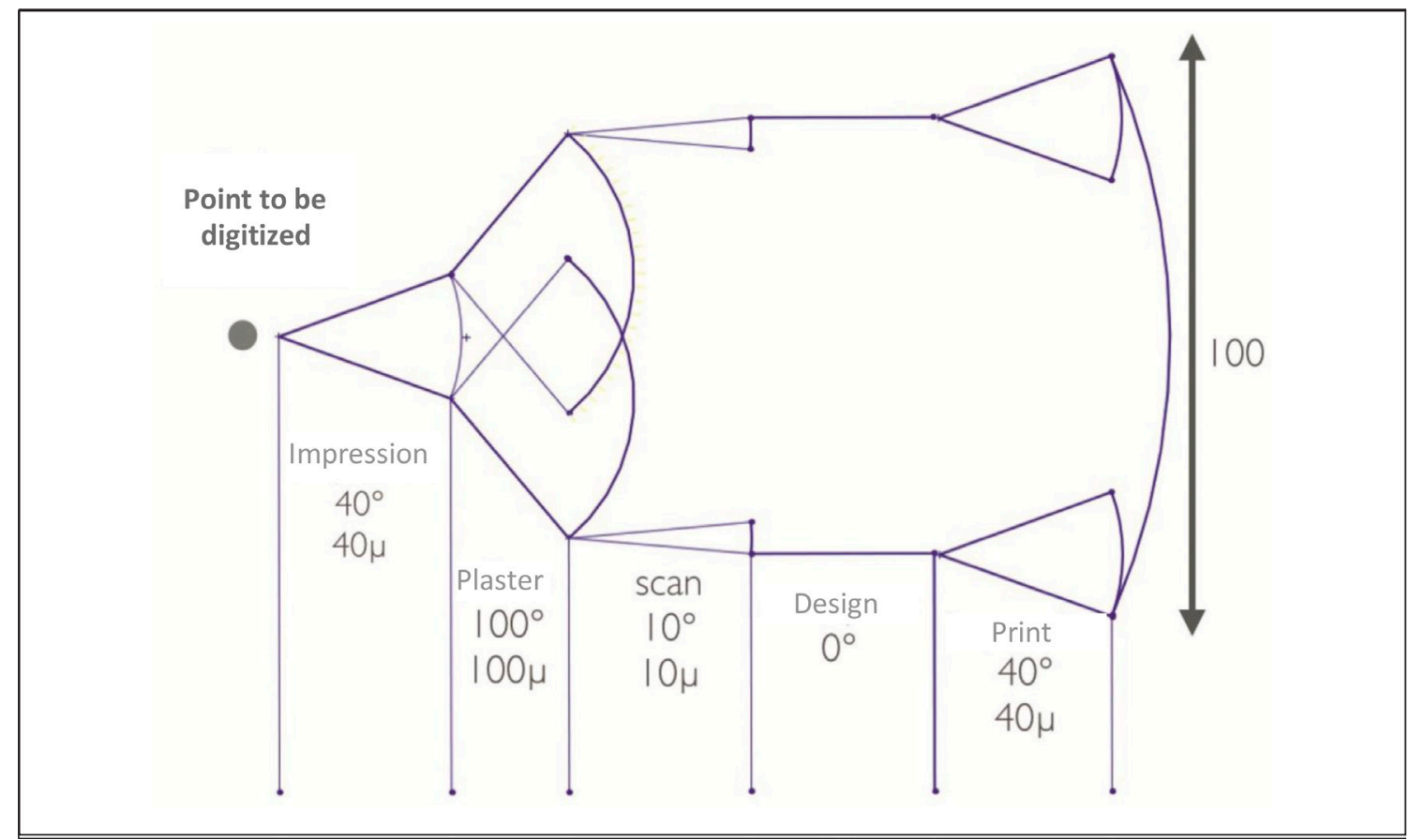

Figure 1

Scanned plaster model.

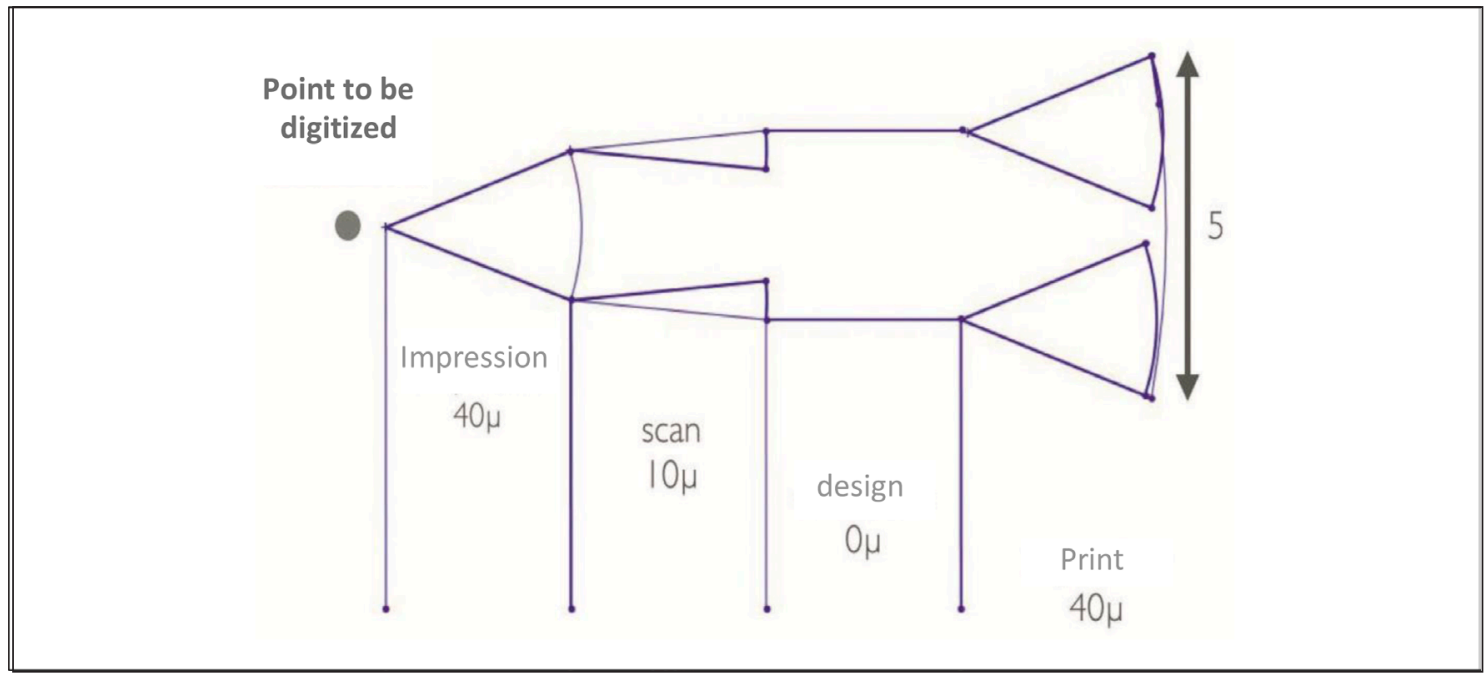

Figure 2

Impression scanned directly without casting.

time taken or the number of parts to be created) horizontal or vertical positioning, with the support frames according-
Iy placed. It should be mentioned that the cross-selling strategy of the manufacturers of scanners and printers is to 


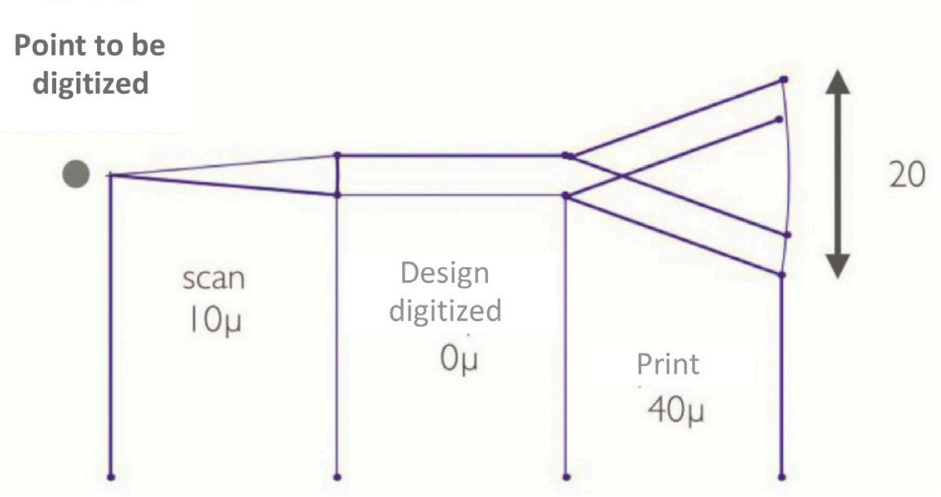

Figure 3

Intraoral optical impression.

offer compatible, adapted, and adaptable software solutions.

It is, therefore, a continuous digital flow, starting from the intraoral scan and ending when the orthosis has been made. Its benefits lie in the reduction of precision errors, the decreased handling of materials, and its ability to be conducted in the lab as a "background task" so that you can work while the printer is working.

If our paper printer provides us with a completed work that is ready to be used, it is not the same for the structure built by the 3D printer.

On the one hand, there may be minimal manipulation to eliminate

\section{USES IN DFO}

The precision of the work conducted seems to assist in the improvement of our treatments. Even if the cuspfossa tripod contact at the end of the treatment remains the holy grail, the more precisely the teeth are placed in the rest of the supports which have been detached from the part. However, it is necessary to make this part biocompatible and adapted to its intended use. There is a specific post-processing phase for each resin, validated by the manufacturers so as to eliminate the surplus of unpolymerized resin, on extricating the part from the liquid bath. This is necessary to minimize precipitation crystallization.

The packaging of the produced part does not differ from the classical production process of the orthodontist, from sanitization to shipping and finally tracking.

patients, the lower the risks of relapse and the amount of effort expended.

Continuous digital flow $\mathrm{f}^{15,17}$ is a real advancement which will decrease laboratory delays and improve the accuracy of the work. Moreover, this avoids having to retake an impression if a device 
deteriorates or is lost, which is very helpful in terms of organization. In the case of aligner treatments, practitioners are able, after simple training to use a specific software ${ }^{17}$, to virtually configure the predictive models by themselves.

Practitioners are really prescribers whose role is not limited to sending impressions and to validating the procedure.

Of course, the existing processes have proven their effectiveness and can be repeatedly applied, but the edge that optical impressions have is that during the course of treatment, any delays are considerably decreased should it become necessary to redo an impression or retake an occlusion.

In this regard, there may be several approaches to creating the cleats necessary for this type of treatment.

When all the work is complete, it is customary for the lab to provide an indirect bonding splint for the cleats and first aligners.

Given the precision of the optical impression, it is advisable to conduct a new scan with the cleats in place, which requires extra time in the chair. In fact, the slightest overshoot of the bonding composite is detrimental to the manufacturing accuracy of the aligners, compared to the thermoforming of a PET plate.

In addition, the cleats should be designed in CAD using specific engineering software and imported into the preinstalled development software. This allows for either conventional indirect bonding or the design of a direct composite molding system with a vent in the splint to eliminate any excess.

Dental-retaining splints are manufactured using this technique. The benefits have been discussed in detail by Bonafe, Lachiche, Egea, Lhermet, and Canal $^{2}$, in a time where wire-bonded retainers are enjoying less success. Patients are now excited to wear them as they report that they are less awkward than the usual thermoformed splints in terms of adaptation.

Mandibular-positioning guides for orthognathic surgery can also be produced with fewer material constraints because they only stay in the mouth for the duration of the surgical intervention.

\section{LIMITS}

This technique has certain limits, which exist only to be tested. First of all, in orthodontic surgery, the printing of sintered metal is too expensive to be considered practical.

Although printing the working or study models may seem easy, controlling the entire process of manufacturing resin orthotics requires enhanced knowledge of computers and materials. It is this difficulty which sometimes gives priority to thermoforming on printed models; it represents a technique that has already been mastered.

In addition to digital innovation, it is important to keep up to date with research on materials so as to discover new resins, as well as upgrade our engineering capabilities to be able to perfect the printers. 


\section{PERSPECTIVES AND CONCLUSION}

It is undeniable that like any other novelty, continuous digital flow has caused a buzz in DFO, and has aroused the interest of the practitioners (who crave novelty), the orthotists, and the commercial firms.

It is important to bear in mind that this is only a method of producing orthotics. In the development of a diagnosis, nothing will replace the clinical examination of our patient, their oral functions, theirTMJ (temporomandibular joint) and their posture. The specialist will not be replaced by the machine.

With evolution being constant we are working on pairing the image provided by CBCT with the STL file in order to have a virtual representation not only of the teeth but of their roots as well. The benefit is immediate in terms of developing a diagnosis, given that what the practitioner has virtually constructed in his mind by synthesizing the data from the casts and radiological snapshots ${ }^{8}$, becomes visible and manipulable on the screen. It will also allow us to achieve a configuration facilitated by a complete dental visualization, from the apex to occlusal edge. One of the main applications is the printing of surgical guides used in the implantation of mini-screw anchorages, thereby considerably decreasing the risk of root perforation ${ }^{13}$.

Further advances can be achieved, with the modelling of the teeth we can now consider the possibility of predicting orthodontic movement, taking into account the "coefficient of friction" in the alveolar bone, regardless of the device used. A search algorithm remains to be written in this regard.
The guiding principle of this process is the optimization of our treatments, but there is another application-the production of elastofinishers or positioners. ${ }^{14,11}$ This could be considered as depositing locks which, insofar as the optical impressions are, free from undercuts; the locks are then put in place. A simple optical impression will improve the result of multi-attachment treatment, be it vestibular or lingual. We are waiting for specific resins so as to validate the process.

Soft resin printing will make it possible to produce an individualized functional educational device in rare cases where the preformed apparatuses are not compatible with the dental coronary anatomy of our patient.

There are printers that can print a part with different materials. It is, therefore, possible to imagine a different elasticity within the same apparatus, thereby allowing multiple applications when mobilizing the teeth.

In conclusion, it appears that this continuous digital flow, from the optical impression to the manufacture of orthoses, is a major advancement for the team of practitioners and orthodontists. Orthodontists see their workload being transformed because mastering the operation of the computer tool that controls the production of the device continues to be crucial to the entire process. This workload is more oriented toward production and engineering while decreasing manual manipulation.

This type of approach allows us to individualize our treatments with the constant concern of improving the results for our patients' benefit. In this way, while keeping abreast of technological 
innovation, we can continue to develop new applications.
Conflict of interest:The authors have declared that they do not have any conflict of interest.

\section{BIBLIOGRAPHY}

1. Berman B. 3-D printing: The new industrial revolution. Bus Horizon 2012;55(2):155-162.

2. Bonafe I, Lachiche V, Egea JC, Lhermet D, Canal P. Occlusodontie et contention temporaire amovible. Rev Orthop Dento Faciale 2015;49:365-373.

3. Campbell T, Williams C, Ivanova O, Garrett B. Could 3D Printing Change the World. Atlantic Council Reports 2011.

4. Carestream Dental. http://www.carestreamdental.com (consulté 20/03/2016).

5. Digital Impressions: 3M TM True Definition Scanner. http://solutions.3mfrance.fr/ consommables et équipements dentaires/prothèse dentaire/empreinte numérique/ (consulté 20/03/2016).

6. Envisiontec. http://www.envisiontec.com/industries/dental/ (consulté 20/03/2016).

7. Formlabs. http://www.formlabs.com/fr/

8. Hajeer MY, Millet DT, Ayoub AF, Siebert JP. Applications of 3D Imaging in Orthodontics: part I. J Orthod 2004;31(1):62-70.

9. Imprimantes 3D Stratasys. http://www.stratasys.com/3D_printers/

10. Itero: Intra Oral Digital Scanner. http://www.itero.com/ (consulté 20/03/2016).

11. Linoleum E, Flageul F. Logique et cohérence. Thérapeutique orthodontique. Ed Quintessence International, 2015.

12. Lythos Digital Impression System. http://www.ormco.com/products/lythos/ (consulté 20/03/2016).

13. Mer G, Brézulier D, Sorel O. Échecs des ancrages osseux. Orthod Fr 2016;87:67-76.

14. Rollet D, Graindorge JC, Guezennec P. Un concept nouveau : I'élastodontie. Rev Orthop Dento Faciale 1991;25(2):149-167.

15. Taneva E, Kusnoto B, Evans CA. 3D Scanning, Imaging and Printing in Orthodontics. http://dx.doi. org/10.5772/60010

16. 3D Systems. http://www.3dsystems.com/ (consulté 20/03/2016).

17. 3 Shape. http://3shapedental.com/orthodontics.aspx?lang=fr/ (consulté 20/03/2016). 